\title{
On new generalizations of Smarzewski's fixed point theorem
}

Wei-Shih Du*

\section{"Correspondence:}

wsdu@nknucc.nknu.edu.tw

Department of Mathematics,

National Kaohsiung Normal

University, Kaohsiung, 824, Taiwan

\begin{abstract}
In this work, we prove some new generalizations of Smarzewski's fixed point theorem and some new fixed point theorems which are original and quite different from the well-known results in the literature.

MSC: 46B20; 47H09; 54H 25

Keywords: strictly convex Banach space; uniformly convex Banach space; uniformly convex in every direction (UCED); $\lambda$-firmly nonexpansive mapping; asymptotic radius; asymptotic center; (UAC)-property; reactive firmly nonexpansive mapping;

Smarzewski's fixed point theorem
\end{abstract}

\section{Introduction and preliminaries}

Let $(X,\|\cdot\|)$ be a normed space with its zero vector $\theta$. We use $B(X)$ and $S(X)$ to denote respectively the closed unit ball and unit sphere centered at $\theta$ with radius 1 , that is,

$$
B(X)=\{x \in X:\|x\| \leq 1\}
$$

and

$$
S(X)=\{x \in X:\|x\|=1\} .
$$

The notion of uniformly convex ( $U C$, for short) Banach space was introduced by Clarkson [1], and the research of geometric properties of the Banach space started from 1936. The function $\delta_{X}:[0,2] \rightarrow[0,1]$, defined by

$$
\delta_{X}(\varepsilon)=\inf \left\{1-\left\|\frac{x+y}{2}\right\|: x, y \in B(X),\|x-y\| \geq \varepsilon\right\} \quad \text { for } \varepsilon \in[0,2],
$$

is called the modulus of convexity of $X$. The normed space $X$ is called uniformly convex if $\delta_{X}(\varepsilon)>0$ for every $\varepsilon \in(0,2]$. It is well known that a uniformly convex Banach space is reflexive and all Hilbert spaces and Banach spaces $\ell^{p}$ and $L^{p}(1<p<\infty)$ all are uniformly convex; see, e.g., [2-7] for more details. The normed space $X$ is said to be strictly convex if $\|x+y\|<2$ whenever $x, y \in S(X)$ with $\|x-y\|>0$. It is obvious that a Banach space $X$ is strictly convex if and only if $\delta_{X}(2)=1$. It is well known that the strict convexity of a normed space $X$ can be characterized by the properties: for any nonzero vectors $x, y \in X$, if

\section{Springer}

○2014 Du; licensee Springer. This is an Open Access article distributed under the terms of the Creative Commons Attribution License (http://creativecommons.org/licenses/by/2.0), which permits unrestricted use, distribution, and reproduction in any medium, provided the original work is properly cited. 
$\|x+y\|=\|x\|+\|y\|$, then $y=c x$ for some real $c>0$. For each $\varepsilon>0$, the modulus of convexity of $X$ in the direction $z \in S(X)$ is defined by

$$
\delta_{X}(\varepsilon, z)=\inf \left\{1-\left\|\frac{x+y}{2}\right\|: x, y \in B(X), x-y=\lambda z,|\lambda| \geq \varepsilon\right\} .
$$

Clearly, $\delta_{X}(\varepsilon)=\inf \left\{\delta_{X}(\varepsilon, z): z \in S(X)\right\}$. The Banach space $X$ is called uniformly convex in every direction (UCED, for short) if for any $z \in S(X)$ and $\varepsilon>0, \delta(\varepsilon, z)>0$. Some characterizations of $U C E D$ Banach spaces were proved by Day et al. [7]; see also [4].

Fact 1.1 (see, e.g., [2-4, 7])

(a) Every $U C$ Banach space is $U C E D$.

(b) Every $U C E D$ Banach space is strictly convex.

Let $(X,\|\cdot\|)$ be a Banach space and $K$ be a given nonempty closed subset of $X$. For $x \in X$ and a bounded sequence $\left\{x_{n}\right\} \subset X$, define the asymptotic radius of $\left\{x_{n}\right\}$ at $x$ as the number

$$
r\left(x,\left\{x_{n}\right\}\right)=\limsup _{n \rightarrow \infty}\left\|x-x_{n}\right\| .
$$

The asymptotic radius of $\left\{x_{n}\right\}$ with respect to $K$ is defined by

$$
r\left(K,\left\{x_{n}\right\}\right)=\inf \left\{r\left(x,\left\{x_{n}\right\}\right): x \in K\right\},
$$

and the set

$$
A\left(K,\left\{x_{n}\right\}\right)=\left\{x \in K: r\left(x,\left\{x_{n}\right\}\right)=r\left(K,\left\{x_{n}\right\}\right)\right\}
$$

is called the asymptotic center of $\left\{x_{n}\right\}$ with respect to $K$. For any bounded sequence $\left\{x_{n}\right\}$ in $X, r\left(x,\left\{x_{n}\right\}\right)$ is easily seen to be a nonnegative, continuous and convex functional of $x \in X$. Moreover, if $K$ is a nonempty convex subset of $X$, then $A\left(K,\left\{x_{n}\right\}\right)$ is also convex.

Fact 1.2 [8, Lemma 2.2] Every bounded sequence in a UCED Banach space $X$ has a unique asymptotic center with respect to any nonempty weakly compact convex subset of $X$.

Definition 1.1 A normed space $(X,\|\cdot\|)$ is said to have the $(U A C)$-property if every bounded sequence in $X$ has a unique asymptotic center with respect to any nonempty weakly compact convex subset of $X$.

According to Facts 1.1 and 1.2, it is easy to know that Hilbert spaces, $U C$ Banach spaces and $U C E D$ Banach spaces all have the $(U A C)$-property.

Let $C$ be a nonempty subset of a normed space $(X,\|\cdot\|)$ and $T: C \rightarrow X$ be a mapping. $T$ is said to be nonexpansive if

$$
\|T x-T y\| \leq\|x-y\| \quad \text { for all } x, y \in C .
$$

The concept of firmly nonexpansive mappings was introduced by Bruck [9]. Let $\lambda \in(0,1)$. The mapping $T$ is said to be $\lambda$-firmly nonexpansive [9] if

$$
\|T x-T y\| \leq\|(1-\lambda)(x-y)+\lambda(T x-T y)\| \quad \text { for all } x, y \in C .
$$


It is obvious that every $\lambda$-firmly nonexpansive mapping is nonexpansive, but the converse is not true. The following example shows that there exists a nonexpansive mapping which is not a $\lambda$-firmly nonexpansive mapping for some $\lambda \in(0,1)$.

Example A Let $X=\mathbb{R}$ with the absolute-value norm $|\cdot|$ and $C=[2,10]$. Let $T: C \rightarrow X$ be defined by $T x=-x$. Then $T$ is a nonexpansive mapping. For $x=6, y=4$ and $\lambda=\frac{1}{2}$, we have

$$
|T(x)-T(y)|=2>0=|(1-\lambda)(x-y)+\lambda(T(x)-T(y))|,
$$

which deduces that $T$ is not a $\frac{1}{2}$-firmly nonexpansive mapping. In fact, $T$ is not $\lambda$-firmly nonexpansive for all $\lambda \in(0,1)$.

In 1965, Browder [10], Kirk [11] and Göhde [12] proved respectively that every nonexpansive mapping $T$ from a nonempty weakly compact convex subset $K$ of a uniformly convex Banach space $X$ into itself has a fixed point. It is know that the convexity of sets and mappings plays an important role in fixed point theory and the union of convex sets does not ensure that it is convex. In 1991, Smarzewski [13] proved the following interesting theorem.

Theorem 1.1 (Smarzewski [13]) Let $X$ be a uniformly convex Banach space and $C=$ $\bigcup_{k=1}^{n} C_{k}$ be a finite union of nonempty weakly compact convex subsets $C_{k}$ of $X$. If $T: C \rightarrow C$ is a $\lambda$-firmly nonexpansive mapping for some $\lambda \in(0,1)$, then $T$ has a fixed point in $C$.

Smarzewski's fixed point theorem (i.e., Theorem 1.1) is not always true if $T$ is merely nonexpansive, even in $X=\mathbb{R}$.

Example B [13] Let $X=\mathbb{R}$ with the absolute-value norm $|\cdot|$ and $C=[-2,-1] \cup[-2,-1]$. Then the mapping $T: C \rightarrow C$ defined by $T x=-x$ is nonexpansive and fixed point free.

In this paper, in order to promote Smarzewski's fixed point theorem, we first introduce the concept of reactive firmly nonexpansive mappings.

Definition 1.2 Let $C$ be a nonempty subset of a normed space $(X,\|\cdot\|)$ and $\varphi: C \times C \rightarrow$ $[0,1)$ be a function. A mapping $T: C \rightarrow X$ is said to be reactive firmly nonexpansive with respect to $\varphi$ if

$$
\|T x-T y\| \leq\|(1-\varphi(x, y))(x-y)+\varphi(x, y)(T x-T y)\| \quad \text { for all } x, y \in C .
$$

\section{Remark 1.1}

(a) Every reactive firmly nonexpansive mapping is nonexpansive.

(b) It is obvious that any $\lambda$-firmly nonexpansive mapping is reactive firmly nonexpansive with respect to the function $\varphi$ defined by $\varphi(s, t)=\lambda$ for all $(s, t) \in C \times C$.

Example $\mathbf{C}$ Let $X=\mathbb{R}$ with the absolute-value norm $|\cdot|$ and $C=[2,5] \cup[10,20]$. Let $T: C \rightarrow X$ be defined by

$$
T x:= \begin{cases}-\frac{3}{5} x, & \text { if } x \in[2,5], \\ -\frac{1}{2} x, & \text { if } x \in[10,20] .\end{cases}
$$


Then the following statements hold.

(a) $T$ is a nonexpansive mapping.

(b) $T$ is not $\frac{1}{3}$-firmly nonexpansive.

(c) Define $\varphi: C \times C \rightarrow[0,1)$ by

$$
\varphi(s, t):= \begin{cases}\frac{1}{10}, & \text { if } s, t \in[2,5] \\ \frac{1}{3}, & \text { otherwise. }\end{cases}
$$

Then $T$ is reactive firmly nonexpansive with respect to $\varphi$.

Proof Obviously, statement (a) holds. To see (b), let $x=4$ and $y=3$. Since

$$
|T(x)-T(y)|=\frac{3}{5}>\frac{7}{15}=\left|\left(1-\frac{1}{3}\right)(x-y)+\frac{1}{3}(T x-T y)\right|,
$$

we show that $T$ is not $\frac{1}{3}$-firmly nonexpansive. Finally, we prove (c). We consider the following four possible cases to verify

$$
|T x-T y| \leq|(1-\varphi(x, y))(x-y)+\varphi(x, y)(T x-T y)|
$$

for all $x, y \in C$.

Case 1. If $x, y \in[2,5]$, then

$$
|T(x)-T(y)|=\frac{3}{5}|x-y|
$$

and

$$
\begin{aligned}
|(1-\varphi(x, y))(x-y)+\varphi(x, y)(T x-T y)| & =\left|\left(1-\frac{1}{10}\right)(x-y)+\frac{1}{10}(T x-T y)\right| \\
& =\frac{21}{25}|x-y| .
\end{aligned}
$$

So (1.1) holds for all $x, y \in[2,5]$.

Case 2. If $x \in[2,5]$ and $y \in[10,20]$, then

$$
|T(x)-T(y)|=\frac{1}{2} y-\frac{3}{5} x
$$

and

$$
\begin{aligned}
|(1-\varphi(x, y))(x-y)+\varphi(x, y)(T x-T y)| & =\left|\left(1-\frac{1}{3}\right)(x-y)+\frac{1}{3}(T x-T y)\right| \\
& =\frac{1}{2} y-\frac{7}{15} x .
\end{aligned}
$$

Since

$$
\left(\frac{1}{2} y-\frac{7}{15} x\right)-\left(\frac{1}{2} y-\frac{3}{5} x\right)=\frac{2}{15} x>0,
$$

we prove that (1.1) holds for all $x \in[2,5]$ and $y \in[10,20]$. 
Case 3. If $x \in[10,20]$ and $y \in[2,5]$, then

$$
|T(x)-T(y)|=\frac{1}{2} x-\frac{3}{5} y
$$

and

$$
|(1-\varphi(x, y))(x-y)+\varphi(x, y)(T x-T y)|=\frac{1}{2} x-\frac{7}{15} y .
$$

Since

$$
\left(\frac{1}{2} x-\frac{7}{15} y\right)-\left(\frac{1}{2} x-\frac{3}{5} y\right)=\frac{2}{15} y>0
$$

we prove that (1.1) holds for all $x \in[10,20]$ and $y \in[2,5]$.

Case 4. If $x, y \in[10,20]$, then

$$
|T(x)-T(y)|=\frac{1}{2}|x-y|
$$

and

$$
\begin{aligned}
|(1-\varphi(x, y))(x-y)+\varphi(x, y)(T x-T y)| & =\left|\left(1-\frac{1}{3}\right)(x-y)+\frac{1}{3}(T x-T y)\right| \\
& =\frac{1}{2}|x-y| .
\end{aligned}
$$

So (1.1) holds for all $x, y \in[10,20]$.

By Cases 1-4, we verify that inequality (1.1) holds for all $x, y \in C$. Hence $T$ is reactive firmly nonexpansive with respect to $\varphi$ and (c) is proved.

In this paper, we establish some generalizations of Smarzewski's fixed point theorem for reactive firmly nonexpansive mappings and some new fixed point theorems which are original and quite different from the well-known results in the literature.

\section{New generalizations of Smarzewski's fixed point theorem and applications to fixed point theory}

In this section, we first establish a new fixed point theorem for reactive firmly nonexpansive mappings which is generalized Smarzewski's fixed point theorem. We assume $0<\varphi(s, t)<1$ for all $(s, t) \in C \times C$ in the following main theorem.

Theorem 2.1 Let $X$ be a strictly convex Banach space with its zero vector $\theta$ and $C=$ $\bigcup_{k=1}^{n} C_{k}$ be a finite union of nonempty weakly compact convex subsets $C_{k}$ of $X$. Let $\varphi$ : $C \times C \rightarrow(0,1)$ be a function and $T: C \rightarrow C$ be a mapping. Suppose that

(a) $X$ has the (UAC)-property,

(b) $T$ is reactive firmly nonexpansive with respect to $\varphi$.

Then $T$ has a fixed point in $C$.

Proof Let $z \in C$ be given. Since $C$ is $T$-invariant and $C$ is bounded, the sequence $\left\{T^{j} z\right\}_{j=1}^{\infty} \subset$ $C$ is bounded. Define the functional $r: X \rightarrow[0, \infty)$, the asymptotic radius of $\left\{T^{j} z\right\}$ at $x \in X$, 
by

$$
r(x):=r\left(x,\left\{T^{j} z\right\}\right)=\limsup _{j \rightarrow \infty}\left\|x-T^{j} z\right\| .
$$

For each $1 \leq k \leq n$, let the number

$$
r\left(C_{k}\right):=r\left(C_{k},\left\{T^{j} z\right\}\right)=\inf \left\{r(x): x \in C_{k}\right\}
$$

and the set

$$
A\left(C_{k}\right):=A\left(C_{k},\left\{T^{j} z\right\}\right)=\left\{x \in C_{k}: r(x)=r\left(C_{k}\right)\right\}
$$

be respectively the asymptotic radius and the asymptotic center of the sequence $\left\{T^{j} z\right\}$ with respect to $C_{k}$. Since $X$ has the $(U A C)$-property, let $x_{k} \in C_{k}$ be the unique asymptotic center of $\left\{T^{j} z\right\}$ with respect to $C_{k}$ for $1 \leq k \leq n$. So

$$
r\left(x_{k}\right)=r\left(C_{k}\right)=\inf \left\{r(x): x \in C_{k}\right\} \quad \text { for each } 1 \leq k \leq n .
$$

For any $k$ and $j$, since $T$ is nonexpansive, we have

$$
\left\|T x_{k}-T^{j} z\right\| \leq\left\|x_{k}-T^{j-1} z\right\|,
$$

which implies

$$
r\left(T x_{k}\right)=\limsup _{j \rightarrow \infty}\left\|T x_{k}-T^{j} z\right\| \leq \limsup _{j \rightarrow \infty}\left\|x_{k}-T^{j-1} z\right\|=r\left(x_{k}\right) \quad \text { for all } k .
$$

Let

$$
m=\min \left\{r\left(x_{k}\right): 1 \leq k \leq n\right\}
$$

Clearly, $m<\infty$. For arbitrary $x \in C=\bigcup_{k=1}^{n} C_{k}, x \in C_{k_{x}}$ for some $k_{x}, 1 \leq k_{x} \leq n$. Thus we have

$$
r(x) \geq r\left(C_{k}\right)=r\left(x_{k}\right) \geq m .
$$

Taking the infimum for $x$ over $C$ yields $r\left(C,\left\{T^{j} z\right\}\right) \geq m$. Conversely, suppose $r\left(x_{k_{0}}\right)=m$ for some $k_{0}, 1 \leq k_{0} \leq n$. Then

$$
m=r\left(x_{k_{0}}\right) \geq \inf _{x \in C} r(x)=r\left(C,\left\{T^{j} z\right\}\right)
$$

By (2.2) and (2.3), we prove

$$
r\left(C,\left\{T^{j} z\right\}\right)=m
$$

Put

$$
L=\left\{x_{k}: r\left(x_{k}\right)=m\right\} .
$$


It is obvious that $\sharp(L) \leq n$, where $\sharp(L)$ is the cardinal number of $L$. We claim that for $u \in C$ with $r(u)=m$ if and only if $u \in L$. Indeed, it suffices to show that if $u \in C$ with $r(u)=m$, then $u \in L$. Let $u \in C=\bigcup_{k=1}^{n} C_{k}$. Then there is some $k_{u}, 1 \leq k_{u} \leq n$, such that $u \in C_{k_{u}}$. So we obtain

$$
r(u)=m \leq r\left(x_{k_{u}}\right) \leq r(u)
$$

and hence

$$
r(u)=m=r\left(x_{k_{u}}\right) .
$$

By the uniqueness of asymptotic center $x_{k_{u}}$, we have $u=x_{k_{u}}$, which means that $u \in L$.

Next, we will prove $T L \subset L$ (i.e., $L$ is $T$-invariant). For any $u \in L$, since $r(u)=m$, from (2.1) and (2.4) it follows that

$$
m=r(u) \geq r(T u) \geq \inf _{x \in C} r(x)=r\left(C,\left\{T^{j} z\right\}\right)=m
$$

and hence $r(T u)=m$. According to our claim, we get $T u \in L$, which completes the assertion. Now, we show that $T$ has a fixed point in $L$. Suppose to the contrary that $T$ has no fixed point in $L$, that is, $T u \neq u$ for all $u \in L$. Then $T x_{k} \notin C_{k}$ for all $x_{k} \in L$. Indeed, if $T x_{k} \in C_{k}$ for some $x_{k} \in L$, since

$$
m=r\left(x_{k}\right) \geq r\left(T x_{k}\right) \geq \inf _{x \in C_{k}} r(x)=r\left(x_{k}\right)=m,
$$

and by the uniqueness of asymptotic center $x_{k}$, we obtain $T x_{k}=x_{k}$. This means that $x_{k}$ is a fixed point for $T$, contradicting our assumption, hence $T x_{k} \notin C_{k}$ for all $x_{k} \in L$. For $T L \subset L$, there exists $w \in L$ such that $T^{\alpha} w=w$ for some $1 \leq \alpha \leq \sharp(L)$. Since

$$
0 \neq\|w-T w\|=\left\|T^{\alpha} w-T^{\alpha+1} w\right\| \leq\left\|T^{\alpha-1} w-T^{\alpha} w\right\| \leq \cdots \leq\left\|T w-T^{2} w\right\| \leq\|w-T w\|,
$$

it follows that

$$
0<\xi:=\|w-T w\|=\left\|T w-T^{2} w\right\|=\cdots=\left\|T^{\alpha-1} w-T^{\alpha} w\right\|=\left\|T^{\alpha} w-T^{\alpha+1} w\right\| .
$$

Since $T$ is reactive firmly nonexpansive with respect to $\varphi$, we have

$$
\begin{aligned}
\xi & =\left\|T^{i} w-T^{i+1} w\right\| \\
& \leq\left\|\left(1-\varphi\left(T^{i-1} w, T^{i} w\right)\right)\left(T^{i-1} w-T^{i} w\right)+\varphi\left(T^{i-1} w, T^{i} w\right)\left(T^{i} w-T^{i+1} w\right)\right\| \\
& \leq\left(1-\varphi\left(T^{i-1} w, T^{i} w\right)\right)\left\|T^{i-1} w-T^{i} w\right\|+\varphi\left(T^{i-1} w, T^{i} w\right)\left\|T^{i} w-T^{i+1} w\right\| \\
& =\xi
\end{aligned}
$$

for $1 \leq i \leq \alpha$, where $T^{0}=I$ (the identity mapping). Therefore, in view of strict convexity of the norm, there is $t_{i}>0$ such that

$$
T^{i-1} w-T^{i} w=t_{i}\left(T^{i} w-T^{i+1} w\right) \quad \text { for } 1 \leq i \leq \alpha
$$


From (2.5) and (2.6), we have

$$
\xi=\left\|T^{i-1} w-T^{i} w\right\|=t_{i}\left\|T^{i} w-T^{i+1} w\right\|=t_{i} \xi,
$$

which implies that $t_{i}=1$ for all $i$. So, by (2.6) again, we get

$$
\theta \neq v:=w-T w=T w-T^{2} w=\cdots=T^{\alpha-1} w-T^{\alpha} w
$$

Since

$$
T^{\alpha} w=T^{\alpha-1} w-v=\left(T^{\alpha-2} w-v\right)-v=T^{\alpha-2} w-2 v=\cdots=w-\alpha v,
$$

we obtain

$$
w=T^{\alpha} w=w-\alpha v,
$$

which implies $v=\theta$, contradicting the fact that $v \neq \theta$. Therefore $T$ must have a fixed point in $L \subset C$ and this completes the proof.

The following results are immediate consequences of Theorem 2.1.

Corollary 2.1 Let $X$ be a strictly convex Banach space with its zero vector $\theta$ and $C=$ $\bigcup_{k=1}^{n} C_{k}$ be a finite union of nonempty weakly compact convex subsets $C_{k}$ of $X$. Let $T: C \rightarrow$ $C$ be a mapping. Suppose that

(a) $X$ has the (UAC)-property,

(b) $T$ is $\lambda$-firmly nonexpansive for some $\lambda \in(0,1)$.

Then $T$ has a fixed point in $C$.

Corollary 2.2 Let $C=\bigcup_{k=1}^{n} C_{k}$ be a finite union of nonempty weakly compact convex subsets $C_{k}$ of a UCED Banach space $X$ and $\varphi: C \times C \rightarrow(0,1)$ be a function. If $T: C \rightarrow C$ is $T$ is reactive firmly nonexpansive with respect to $\varphi$, then $T$ has a fixed point in $C$.

Corollary 2.3 [8, Theorem 2.8] Let $C=\bigcup_{k=1}^{n} C_{k}$ be a finite union of nonempty weakly compact convex subsets $C_{k}$ of a UCED Banach space $X$. If $T: C \rightarrow C$ is $\lambda$-firmly nonexpansive for some $\lambda \in(0,1)$, then $T$ has a fixed point in $C$.

Remark 2.1 Theorem 2.1 and Corollaries 2.1 and 2.2 all generalize and improve Smarzewski's fixed point theorem, [8, Theorem 2.8] and [14, Theorems 2.8, 2.9].

In Theorem 2.1, if $C_{1}=C_{2}=\cdots=C_{n}:=C$, then we obtain the following new fixed point theorem.

Theorem 2.2 Let $X$ be a strictly convex Banach space with its zero vector $\theta$ and $C$ be $a$ nonempty weakly compact convex subset of $X$. Let $\varphi: C \times C \rightarrow(0,1)$ be a function and $T: C \rightarrow C$ be a mapping. Suppose that

(a) $X$ has the (UAC)-property,

(b) $T$ is reactive firmly nonexpansive with respect to $\varphi$.

Then $T$ has a fixed point in $C$. 
The following results are immediate from Theorem 2.2.

Corollary 2.4 Let $X$ be a strictly convex Banach space with its zero vector $\theta$ and $C$ be a nonempty weakly compact convex subset of $X$. Let $\varphi: C \times C \rightarrow(0,1)$ be a function and $T: C \rightarrow C$ be a mapping. Suppose that

(a) $X$ has the (UAC)-property,

(b) $T$ is $\lambda$-firmly nonexpansive for some $\lambda \in(0,1)$.

Then $T$ has a fixed point in $C$.

Corollary 2.5 Let $C$ be a nonempty weakly compact convex subset of a UCED Banach space $X$. Let $\varphi: C \times C \rightarrow(0,1)$ be a function. If $T: C \rightarrow C$ is reactive firmly nonexpansive with respect to $\varphi$, then $T$ has a fixed point in $C$.

Corollary 2.6 Let $C$ be a nonempty weakly compact convex subset of a UCED Banach space $X$. If $T: C \rightarrow C$ is $\lambda$-firmly nonexpansive for some $\lambda \in(0,1)$, then $T$ has a fixed point in $C$.

Definition 2.1 Let $C$ be a nonempty subset of a normed space $(X,\|\cdot\|)$ and $\alpha, \beta \in(0,1)$. A mapping $T: C \rightarrow X$ is said to be $(\alpha, \beta)$-firmly nonexpansive if

$$
\|T x-T y\| \leq\|(1-\beta)[(1-\alpha)(x-y)+\alpha(T x-T y)]+\beta(T x-T y)\| \quad \text { for all } x, y \in C .
$$

Finally, by applying Theorem 2.1, we give some new fixed point theorems for $(\alpha, \beta)$ firmly nonexpansive mappings.

Theorem 2.3 Let $X$ be a strictly convex Banach space with its zero vector $\theta$ and $C=$ $\bigcup_{k=1}^{n} C_{k}$ be a finite union of nonempty weakly compact convex subsets $C_{k}$ of $X$. Let $T: C \rightarrow$ $C$ be a mapping and $\alpha, \beta$ be positive real numbers satisfying $0<(1-\alpha)(1-\beta)<1$. Suppose that

(a) $X$ has the (UAC)-property,

(b) $T$ is $(\alpha, \beta)$-firmly nonexpansive.

Then $T$ has a fixed point in $C$.

Proof Since $\alpha, \beta>0$ and $0<(1-\alpha)(1-\beta)<1$, we have $\alpha+\beta-\alpha \beta \in(0,1)$. Define $\varphi$ : $C \times C \rightarrow(0,1)$ by

$$
\varphi(s, t):=\alpha+\beta-\alpha \beta \quad \text { for }(s, t) \in C \times C .
$$

Due to $T$ is $(\alpha, \beta)$-firmly nonexpansive, we obtain

$$
\begin{aligned}
\|T x-T y\| & \leq\|(1-\beta)[(1-\alpha)(x-y)+\alpha(T x-T y)]+\beta(T x-T y)\| \\
& =\|(1-\varphi(x, y))(x-y)+\varphi(x, y)(T x-T y)\|
\end{aligned}
$$

for all $x, y \in C$. So, $T$ is reactive firmly nonexpansive with respect to $\varphi$. Therefore the conclusion follows from Theorem 2.1. 
Corollary 2.7 Let $C$ be a nonempty weakly compact convex subset of a UCED Banach space $X$. Let $\alpha, \beta$ be positive real numbers satisfying $0<(1-\alpha)(1-\beta)<1$. If $T: C \rightarrow C$ is $(\alpha, \beta)$-firmly nonexpansive, then $T$ has a fixed point in $C$.

Corollary 2.8 Let $C$ be a nonempty weakly compact convex subset of a uniformly convex Banach space X. Let $\alpha, \beta$ be positive real numbers satisfying $0<(1-\alpha)(1-\beta)<1$. If T : $C \rightarrow C$ is $(\alpha, \beta)$-firmly nonexpansive, then $T$ has a fixed point in $C$.

\section{Competing interests}

The author declares that he has no competing interests.

\section{Acknowledgements}

The author was supported by Grant No. MOST 103-2115-M-017-001 of the Ministry of Science and Technology of the Republic of China.

\section{Received: 22 September 2014 Accepted: 25 November 2014 Published: 12 Dec 2014}

\section{References}

1. Clarkson, JA: Uniformly convex spaces. Trans. Am. Math. Soc. 40, 296-414 (1936)

2. Goebel, K, Reich, S: Uniform Convexity, Hyperbolic Geometry, and Nonexpansive Mappings. Dekker, New York (1984)

3. Goebel, K, Kirk, WA: Topics in Metric Fixed Point Theory. Cambridge University Press, Cambridge (1990)

4. Megginson, RE: An Introduction to Banach Space Theory. Springer, New York (1998)

5. Takahashi, W: Nonlinear Functional Analysis. Fixed Point Theory and Its Applications. Yokohama Publishers, Yokohama (2000)

6. Khamsi, MA, Kirk, WA: An Introduction to Metric Spaces and Fixed Point Theory. Wiley, New York (2001)

7. Day, MM, James, RC, Swaminathan, S: Normed linear spaces that are uniformly convex in every direction. Can. J. Math. 23, 1051-1059 (1971)

8. Du, W-S, Huang, Y-Y, Yen, C-L: Fixed point theorems for nonexpansive mappings on nonconvex sets in UCED Banach spaces. Int. J. Math. Math. Sci. 31, 251-257 (2002)

9. Bruck, RE: Nonexpansive projections on subsets of Banach spaces. Pac. J. Math. 47, 341-355 (1973)

10. Browder, FE: Nonexpansive nonlinear operators in a Banach space. Proc. Natl. Acad. Sci. USA 54, 1041-1044 (1965)

11. Kirk, WA: A fixed point theorem for mappings which do not increase distances. Am. Math. Mon. 72, 1004-1006 (1965)

12. Göhde, D: Zum Prinzip der kontraktiven Abbildung. Math. Nachr. 30, 251-258 (1965) (in German)

13. Smarzewski, R: On firmly nonexpansive mappings. Proc. Am. Math. Soc. 113, 723-725 (1991)

14. Kaczor, W: Fixed points of asymptotically regular nonexpansive mappings on nonconvex sets. Abstr. Appl. Anal. 2003(2), 83-91 (2003)

10.1186/1029-242X-2014-493

Cite this article as: Du: On new generalizations of Smarzewski's fixed point theorem. Journal of Inequalities and Applications 2014, 2014:493

\section{Submit your manuscript to a SpringerOpen ${ }^{\circ}$ journal and benefit from:}

- Convenient online submission

- Rigorous peer review

- Immediate publication on acceptance

Open access: articles freely available online

- High visibility within the field

- Retaining the copyright to your article 